\title{
Impact of COVID-19 Pandemic on Ovarian Cancer Management: Adjusting to the New Normal
}

This article was published in the following Dove Press journal:

Cancer Management and Research

\author{
Lizbeth Soto Jacome' \\ Sachin Kumar Deshmukh ${ }^{2,3}$ \\ Padmamalini Thulasiraman (iD) \\ Nicolette Paolaungthong \\ Holliday $^{4}$ \\ Seema Singh ${ }^{2,3,5}$ \\ 'Department of Biomedical Sciences, \\ College of Allied Health, University of \\ South Alabama, Mobile, AL 36688, USA; \\ ${ }^{2}$ Cancer Biology Program, Mitchell \\ Cancer Institute, University of South \\ Alabama, Mobile, AL 36604, USA; \\ ${ }^{3}$ Department of Pathology, College of \\ Medicine, University of South Alabama, \\ Mobile, AL 36617, USA; ${ }^{4}$ Department of \\ Obstetrics and Gynecology, University of \\ South Alabama, Mobile, AL 36604, USA; \\ ${ }^{5}$ Department of Biochemistry and \\ Molecular Biology, University of South \\ Alabama, Mobile, AL 36688, USA
}

\begin{abstract}
COVID-19, also known as the coronavirus disease 2019, is caused by the severe acute respiratory syndrome coronavirus 2 (SARS-CoV-2) declared pandemic by the World Health Organization (WHO). As the world faces the coronavirus disease 2019 crisis, the oncology community is being impacted by unprecedented challenges. During this trying time, patients with ovarian cancer (OC) have been affected by a delay in diagnosis, surgery, chemotherapy and radiation treatments, and oncology follow-ups being conducted via telemedicine instead of in-person visits. OC patients and their oncologists are balancing the fears of COVID-19 and cancer treatment with the consequences of delaying cancer care. The delay in treatment care that women with $\mathrm{OC}$ are experiencing has resulted in higher levels of cancer worry, anxiety, and depression. In this article, we succinctly review the impact of the COVID-19 pandemic on the diagnosis and treatment and ongoing clinical trials of OC. We also discuss the psychological effects of COVID-19 on women with OC and alternative therapeutic strategies to limit in-person hospital visits to reduce the spread of the disease, and the impact of COVID-19 on OC patients.
\end{abstract}

Keywords: SARS-CoV-2, COVID-19, ovarian cancer management, pandemic, cancer care delay

\section{Introduction}

Coronavirus Disease 2019 (COVID-19) is a disease caused by a novel coronavirus, severe respiratory syndrome coronavirus 2 (SARS-CoV-2). The first case of COVID-19 was reported in Wuhan, China, in December 2019, and it slowly spread globally. ${ }^{1}$ It was declared a pandemic on March 11, 2020, by the World Health Organization (WHO). ${ }^{2,3}$ To date, more than 65 million confirmed cases of COVID19 had been reported in 213 countries with a death toll of nearly 1.4 million individuals. $^{4}$ The majority of COVID-19 cases (over 14 Million) have been reported in the United States, followed by India (over 9 Million). More than six million cases have been reported in Brazil and placed third on the list. The highest number of deaths ( $>273$ Thousand) due to COVID-19 has also been reported in the United States, and the number keeps rising each day. ${ }^{4}$

Coronaviruses are a large family of viruses that can cause mild to severe respiratory illness symptoms in humans. ${ }^{5-7}$ These viruses are transferred to humans from some non-human vertebrates (cats, camels, and bats) following evolution and contact. $5,7,8$ The transmission of SARS-CoV-2 among humans occurs through direct, indirect, or close contact with the infected person through saliva or respiratory secretions. ${ }^{8,9}$ Typical symptoms of COVID-19 appear between 2-14 days after exposure to the virus and include fever or chills,
Correspondence: Seema Singh Department of Pathology, College of Medicine, University of South Alabama, 1660 Springhill Avenue, Mobile, AL 36604, USA

Tel + I 25I-445-9843

Fax + I 25I-460-6994

Email seemasingh@health.southalabama.edu 
cough, shortness of breath, muscle or body aches, the new loss of taste or smell, and sore throat. ${ }^{5-7,10}$ Severe complications of COVID-19 include acute renal failure, acute respiratory distress syndrome, septic shock, acute respiratory injury, and severe pneumonia..$^{5-7,10}$ It is believed that COVID-19 affects different people in different ways, and the chances of death increase if the infected person has other underlying conditions such as diabetes, asthma, or heart disease. ${ }^{11,12}$

Ovarian cancer (OC) is the most lethal gynecologic malignancy and the fifth leading cause of cancer-related deaths among women in the United States. ${ }^{13}$ American Cancer Society (ACS) initially estimated that approximately 21,750 women would be diagnosed with $\mathrm{OC}$ in 2020, and 13,940 will die of the disease in the United States alone. ${ }^{13}$ However, these initial projections could change, considering the negative impact of COVID-19 on human life and healthcare centers' the functionality. Additionally, sequelae from $\mathrm{OC}$ and its associated treatments can exacerbate the likelihood of getting infected and dying from COVID-19. Cancer patients have compromised immune function due to the disease itself and from anticancer therapy ${ }^{14-16}$ Furthermore, transportation to clinics can involve increased social contact points that make these patients more vulnerable to contracting SAR$\mathrm{CoV}-2$ infection than the general population, who can more effectively quarantine at home. In the following sections, we discuss the impact of COVID-19 on the diagnosis, treatment, and ongoing clinical trials of OC. Moreover, we also will review the psychological effects of COVID-19 on women with OC and the new treatment strategies being implemented to help reduce the impact of COVID-19 on OC patients.

\section{General Impact of COVID-I 9 Pandemic on Factors Affecting Patients}

The COVID-19 pandemic outbreak has caused a generalized fear in people and organizations worldwide as everyone anticipates herd immunity and/or vaccinations that have yet to be approved. Healthcare centers that provide essential health services have also been impacted, which has caused inconveniences to patients. During the pandemic, the primary focus of health centers has shifted to the management of the COVID-19 pandemic. ${ }^{17,18}$ For example, the clinics of Emilia-Romagna, one of the most affected Italian regions for COVID-19, postpone or canceled the OC surgeries. ${ }^{19}$ Moreover, due to the fear of disease spread, most hospitals canceled non-emergent and non-urgent patients' visits until a well-formalized plan to manage patient safety and reduce infection risks was in place. Several South African hospitals postpone or canceled the cancer surgeries during the COVID-19 lockdown. ${ }^{20}$ Many countries' lockdowns to prevent COVID-19 community transmission rates also severely affected the travel and import of healthcare appliances, personal protection equipment, and some essential medicines. Companies could not meet timely requirements due to huge demand and restriction and reduced productivity due to the pandemic. ${ }^{21-23}$ Stringent travel restrictions in India also posed a challenge for many patients to visit the hospital for disease diagnosis and treatment. ${ }^{21-23}$ Additionally, in India most medical facilities are in big cities, and many patients travel great distances to receive cancer therapy and follow-up. ${ }^{22}$ When accounting for travel, food, and accommodations, these factors create significant financial toxicity and barriers due to the COVID-19 pandemic, impacting the follow-up visits in these patients. ${ }^{21,24}$ Collectively, the patients' financial and travel obstacles from the pandemic have significantly impacted treatment decisions ${ }^{25,26}$ and compounded the level of emotional stress that is typically seen with patients undergoing cancer therapy.

\section{Effect of the COVID-I 9 Pandemic on Ovarian Cancer Diagnosis}

Without question, OC diagnosis at an early stage is associated with better survival. ${ }^{27}$ However, most of the OC cases are diagnosed at the advanced stage of the disease, and this late diagnosis significantly influences the clinical outcomes. ${ }^{28}$ Besides a large number of OC patients have metastatic diseases at the time of diagnosis as the earlystage disease is generally asymptomatic. ${ }^{29} \mathrm{OC}$ diagnosis is often confirmed by biopsy or surgery, ${ }^{30}$ which requires a patient to visit a healthcare facility equipped with resources. Typically, a provider evaluates a patient and then schedules imaging to assess further. After this step, there is usually a referral to a specialist upon confirmation of a mass. Due to the increasing incidence of COVID-19 positive patient numbers, women with the symptoms of OC experienced delays in this process. Since most OC symptoms are vague, patients may not appear to have urgent complaints necessitating immediate evaluation, whether in the office or emergency room. Thus, the 
COVID-19 pandemic has further slowed down this workup process due to decreases in access to care. A significant drop in the number of urgent referrals has been noted. Moreover, due to this pandemic's overall progression within the few months following its emergence, clinicians and hospitals are adapting fast to the pandemic crisis and developing a recovery plan to overcome hurdles in patient diagnosis and management.

\section{Effect of the COVID-I 9 Pandemic on the Treatment of Ovarian Cancer Patients}

The Standard of care treatment of OC includes debulking surgery plus platinum/taxane-chemotherapy. ${ }^{31,32}$ Before initiating the treatment of OC patients, and gynecology oncologists arrange to schedule the patient for an inperson office visit to discuss the treatment plan for their care. After that, surgery and other treatment plans are made, and the patient is advised to adhere to the treatment plan for optimal results. Further, a risk assessment strategy that includes age, comorbidities, obesity, and sex is considered before cancer treatment. ${ }^{33}$ Below, we discuss the effect of COVID-19 on in-person patient visits and scheduling for surgery and treatment planning.

\section{Effect of COVID-19 on In-Person Clinic Visits}

Following a confirmed OC diagnosis and the treatment, gynecologic oncologists have limited the frequency of inperson office visits to limit the risk of exposure to the SARS-CoV-2. For local patients who are established, providers recommend that patients continue to undergo the necessary imaging studies and laboratory testing. However, instead of an in-person visit, cancer centers and patients prefer virtual visits via telephone or video conference to discuss the treatment plan after disease diagnosis. For patients farther away, providers recommend that patients complete their initial imaging and laboratory testing at a medical facility in their hometown before having the results forwarded electronically or by mail to the provider. After that, the provider will contact the patient virtually to discuss the preferred treatment plan. A survey conducted by the Collegio Italianodei Primari Oncologi Medici (CIPOMO), an Italian association of head physicians in the oncology department determines that Italy noticed several healthcare changes, including the delay in non-urgent visits and the use of telemedicine post-pandemic. ${ }^{34}$ Face-to-face, patient-physician visits are taking place through telemedicine, and unfortunately, providers have postponed all international patients' visits until further approval from the global health authorities. ${ }^{35}$ After completing the treatment, telemedicine continues to be used as a medium for follow-up surveillance unless an examination is required. ${ }^{35}$

\section{Effect on Ovarian Cancer Surgery and Treatment}

OC surgery involves removing the uterus, cervix, bilateral ovaries and fallopian tubes, the omentum (a large flat adipose tissue layer), and possibly lymph nodes. ${ }^{36}$ Debulking surgery was performed to remove as much visible tumor as possible. Optimal debulking may also require removing a part of the small intestine, portion of the liver, spleen, diaphragm stripping, peritoneal stripping, so in certain situations, neoadjuvant chemotherapy may be safer. OC debulking surgery can be complicated and require a considerable amount of time and a critical care support system. ${ }^{37,38}$ Unfortunately, the COVID-19 pandemic has forced hospitals to postpone several elective surgeries, including many cancer surgeries, to secure the resources for the expected increase number of COVID-19 patients. ${ }^{21,39,40}$ Hospitals are also trying to protect their current patients from getting infections from patient visitors. Women with OC have faced severe disruptions in surgical procedures due to this pandemic. ${ }^{41}$ As per a recent study in the United Kingdom (UK), about $54 \%$ of women with OC reported that their treatment was affected due to COVID-19. ${ }^{42}$ A common reason for this delay is to ensure that the patients are not experiencing any symptoms related to the COVID-19 or have not contacted someone with a confirmed COVID-19 diagnosis or COVID-19 symptoms. Patients are also required to have a nasopharyngeal swab test 48 hours before surgery. In a particular situation, some patients are also being asked to have another swab test on the day of admission before surgery. It is also recommended that if surgery is must, all surgical staff should have adequate protection to reduce the virus's spread. ${ }^{43}$ A national online survey was established by the Italian Society of Gynecology and Obstetrics, and the Multicenter Italian Trials in OC and gynecologic malignancies evaluated the impact of COVID-19 on the clinical activity of gynecologist oncologists and assessed the changes that have been implemented to reduce the risks of COVID-19 infection. ${ }^{44}$ This study suggests that 
out of the 604 participants, 38\% of the gynecologic surgeons expressed concern about the impact of COVID-19 on the treatment. Also, $21 \%$ of the total participants indicated a decrease in the use of laparoscopy. In addition, $20-25 \%$ of the total participants affirmed a decline in the use of radical surgical procedures. ${ }^{44}$ It is suggested that only those procedures that are less demanding should be continued. While more extensive procedures should only be continued on an absolute necessary case. ${ }^{44}$

Neoadjuvant chemotherapy is performed in case of inoperability of OC to reduce the tumor load. Moreover, cytoreductive surgery is beneficial in patients who have limited metastasis. Patients being treated with chemotherapy are also experiencing significant delays. ${ }^{45}$ According to an online survey performed in the USA, about $33 \%$ of women with OC experienced a delay in cancer care due to the COVID-19 outbreak. $^{42}$ This delay in surgery compelled women to receive additional cycles of chemotherapeutic drugs while waiting for surgery. ${ }^{41}$ Further, considering the effect of COVID-19 on the host immune system, cancer immunotherapy treatments are suggested to be used with caution as immune-related adverse events could lead to an abnormal immune response in the case of SAR-CoV-2 infection. ${ }^{46}$ It has also been feared that the surgery and other local and systemic therapies could also be extremely harmful to the patients harboring asymptomatic COVID-19 infection. ${ }^{47}$ Retrospective analyses of clinical data of patients with COVID-19 who underwent elective surgeries from January 1 to February 5, 2020, reported that $44.1 \%$ of patients required ICU admission postoperatively due to COVID-19 related severe symptoms. $^{47}$ Thus, identification of COVID-19 infection before surgery is crucial to minimizing the possible complications. Other than post-surgery complications in patients, there is always a risk of potential contamination of the medical staff working in the surgery room during the procedures. Along with open surgery, laparoscopic procedures could also participate in the possible contamination. ${ }^{43}$ Previous studies on DNA viruses indicate that viral components could be identified in surgical smoke and spread to nearby individuals. ${ }^{48,49}$ During the current pandemic, the cancer research community realized the need for a special center or hubs free of COVID-19 to treat cancer patients. ${ }^{50,51}$ At these hubs, oncologists are supplied with necessary supplies to provide the adequate care that a cancer patient needs. To minimize the risk of virus and reduce the number of postponed procedures,
COVID-19-free cancer hubs are suggested to play a crucial role in cancer care. ${ }^{50}$

\section{Effect of the COVID-I9 Pandemic on Ongoing Clinical Trials}

Clinical trials are conducted to evaluate the efficacy of novel therapeutics or novel treatment approaches to receive Food and Drug Agency (FDA) approval in the USA for clinical use. Clinical trials require several visits following participants' enrollment in trials and include multiple interactions between patients, physicians, and research coordinators. These interactions increase the risk of exposure to SARS-CoV-2, and for this fear, disruption of all types of clinical studies, including cancer-related trials, have been noted. ${ }^{52}$ In the USA alone, the enrollment of new patients for Phase 2 and Phase 3 cancer trials was reported to be decreased by $46 \%$ to $48 \%$ in the last two weeks of March 2020. ${ }^{52}$ An ongoing OC Phase 1 clinical trial (NCT03564340) at Dana-Farber assessing the safety and pharmacokinetics of REGN4018 as monotherapy or in combination with cemiplimab has also been impacted. ${ }^{52}$ REGN4018 is a bispecific antibody that binds both Mucin 16 (MUC16), a glycoprotein which is overexpressed on OC cells, and CD3 that is expressed on $\mathrm{T}$ cells, thus bridging cancer cells with the $\mathrm{CD}^{+} \mathrm{T}$ cells. ${ }^{53}$ The COVID-19 pandemic required that enrolled patients with OC answer a set of questions before each blood draw, each interaction with the physician, and while receiving the infusion. ${ }^{52}$ Enrolled patients in the trial also had to follow the hospital's no-visitor policy. Additionally, the hospital preferred to keep patients' visits shorter with reduced waiting times to avoid unnecessary exposure. ${ }^{52}$

Several new restrictions on clinical trials have been implemented to reduce virus transmission due to ambulatory patient visits. It has been recommended that if a study requires medications that they are supplied by mail to avoid patients picking them up in-person. If during a study, a trial patient tests positive for COVID-19, then the patient should be removed from the study and must follow the recommended guidelines by the institution. For a significant period, restrictions were also put on all the research personnel, including research coordinators, to work from home unless needed for vital sign testing and investigation. $^{54-56}$ These restrictions are slowly being lifted as the hospitals adapt to the new and implemented strategies to contain the virus's spread. 


\section{Alternative Therapeutic Strategies to Limit In-Person Hospital Visits}

Cancer patients who receive chemotherapy often suffer from viral or bacterial infections during therapy due to therapy-induced immune suppression. ${ }^{57,58}$ These infections do not usually modify the standard care of treatment, but in the case of COVID-19 infection, the availability of limited data poses a big challenge for making decisions on continuing the therapy. In some instances, oral treatments are being administered as an alternative to infusion chemotherapies to limit a patient's visit to the infusion clinic. $^{55}$ While this helps decrease the chances of virus spread, patients cannot benefit from a close patientphysician relationship. For the treatment of OC, several classes of drugs such as inhibitors of poly(ADP-ribose) polymerase (PARP), hormonal therapies, and targeted agents can be utilized as alternatives to intravenous therapies. Randomized placebo-controlled trials of PARP inhibitors have demonstrated improved progression-free survival when used as maintenance therapy in both the front line and platinum-sensitive settings. ${ }^{59}$ Notably, a phase 3 study evaluating PARP inhibitors' efficacy against intravenous chemotherapy in patients with BRCA1- or BRCA2-positive and platinum-sensitive relapsed advanced OC suggested that PARP inhibitors can be an alternative to intravenous chemotherapy. ${ }^{60}$ Thus, if a woman with OC does not respond to platinumbased chemotherapy, she may receive an oral PARP inhibitor therapy to maintain the disease in these trying times.

\section{Psychological Impact of a Pandemic on the Treatment of Ovarian Cancer Patients}

The pandemic has impacted the quality of life for women with OC significantly, which is feared to affect their treatment outcomes. ${ }^{42}$ An online survey used the Hospital Anxiety and Depression Scale (HADS) and the Cancer Worry Scale (CWS) to measure women's quality of life with a current or previous OC diagnosis. The survey findings were that about $89 \%$ of women experienced high levels of worry about their cancer during the pandemic. ${ }^{42}$ The different factors associated with high levels of cancer worry included age being less than 65 years, scheduling for cancer treatment or surgery, self-description as being immunocompromised, delay in oncology care, and the use of telemedicine. ${ }^{42}$ The study found that 285 of 555 women suffered from borderline or abnormal anxiety. In addition, the results indicated that 147 of 555 women experienced borderline or abnormal depression.

COVID-19 has also affected the available medical support to patients with OC. In a survey by Target Ovarian Cancer, a UK-based ovarian cancer charity, it was found that about $27 \%$ of women with OC diagnosis faced a considerable change in patient care and support after the pandemic. ${ }^{61}$ A large number $(79 \%)$ of women with $\mathrm{OC}$ were advised to use the shield that restricted these women's participation in the family activities that they enjoy. ${ }^{61}$ These restrictions further increased the social isolation and loneliness that negatively impacted physical and emotional health and wellbeing. Moreover, due to rescheduling or change in cancer treatment, many women face uncertainty, which adds to their cancer diagnosis stress. Further, during these challenging times, access to psychological support is also limited to the patients intensifying their existing worry, anxiety, and depression.

\section{Conclusion and Perspective}

Cancer patients, their families, and healthcare facilities are witnessing unprecedented challenges during the COVID-19 crisis. The COVID-19 crisis has affected OC patients' care, specifically, in-person office visits, laboratory tests, imaging studies, treatments, and surgeries (Figure 1). Clinical trials that were being conducted to help discover new therapies for OC patients have also been interrupted. Overall, patients with OC are experiencing a delay in their cancer care due to federal guidelines and others by the patient's choice or other circumstances. These delays in care have not only caused higher levels of cancer worry, anxiety, and depression in women with OC, but could have adversely affected clinical outcomes if the disease progressed beyond the treatment limits during treatment delay. The COVID-19 pandemic changed cancer care delivery to protect both the cancer patient and the caregiver from the virus infection. Practices, including the use of masks, temperature checks, and self-assessment questionnaires before entering the clinic and social distancing are new standards (Figure 2). To ensure cancer care during this pandemic, COVID-19-free hubs have been established to provide cancer care if necessary. These hubs are virusfree centers where cancer patients can receive the treatment they need while being less exposed to the SARS-CoV-2 infection. Prior to cancer patients entering the hub, they are swabbed for COVID-19 and must test negative. The establishment of these hubs allows patients 


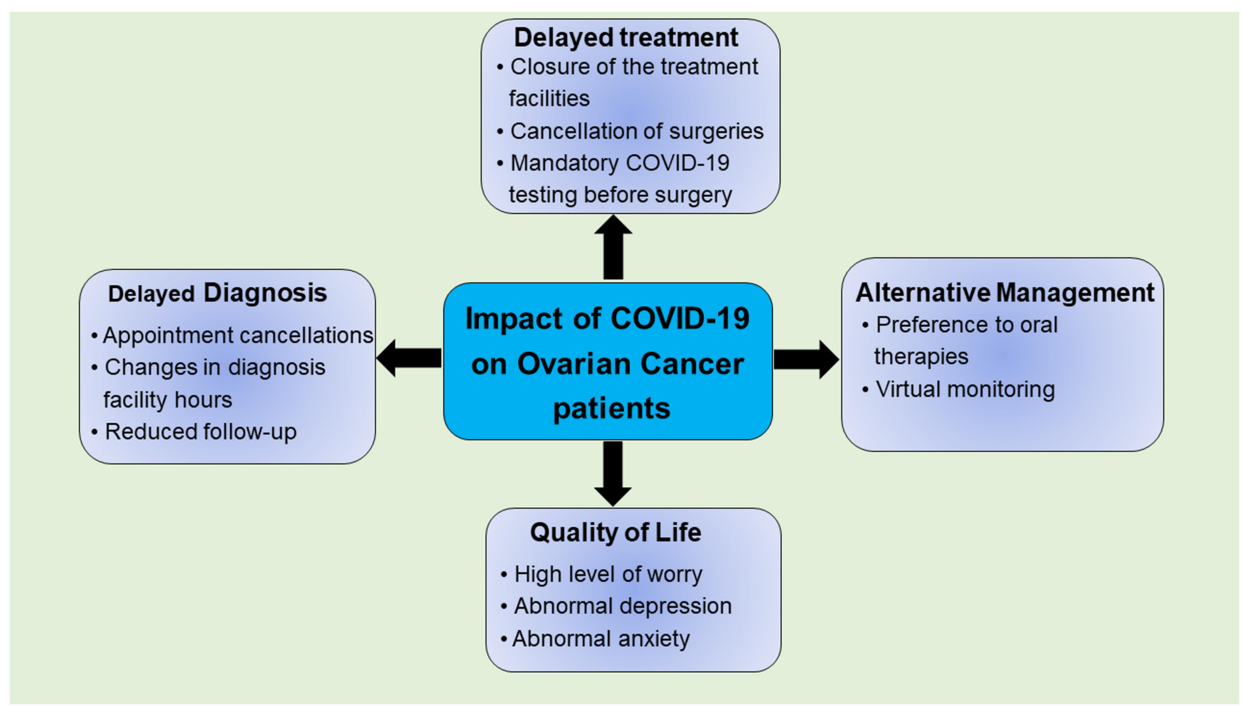

Figure I Impact of COVID-I9 pandemic on patients with ovarian cancer. Hospitals reserved their capacity for handling the flood of COVID-I9 patients during the pandemic that resulted in delays in ovarian cancer diagnosis and treatment. In addition, diagnosis and surgery appointments were canceled. Patients' hesitation to see doctors for a new symptom or routine screening also affected the diagnosis and monitoring. Further, to decrease the chances of virus spread, alternative oral therapeutic strategies were opted to limit in-person hospital visits. Changes in patient care and support contributed to the increased worry, anxiety, and depression.

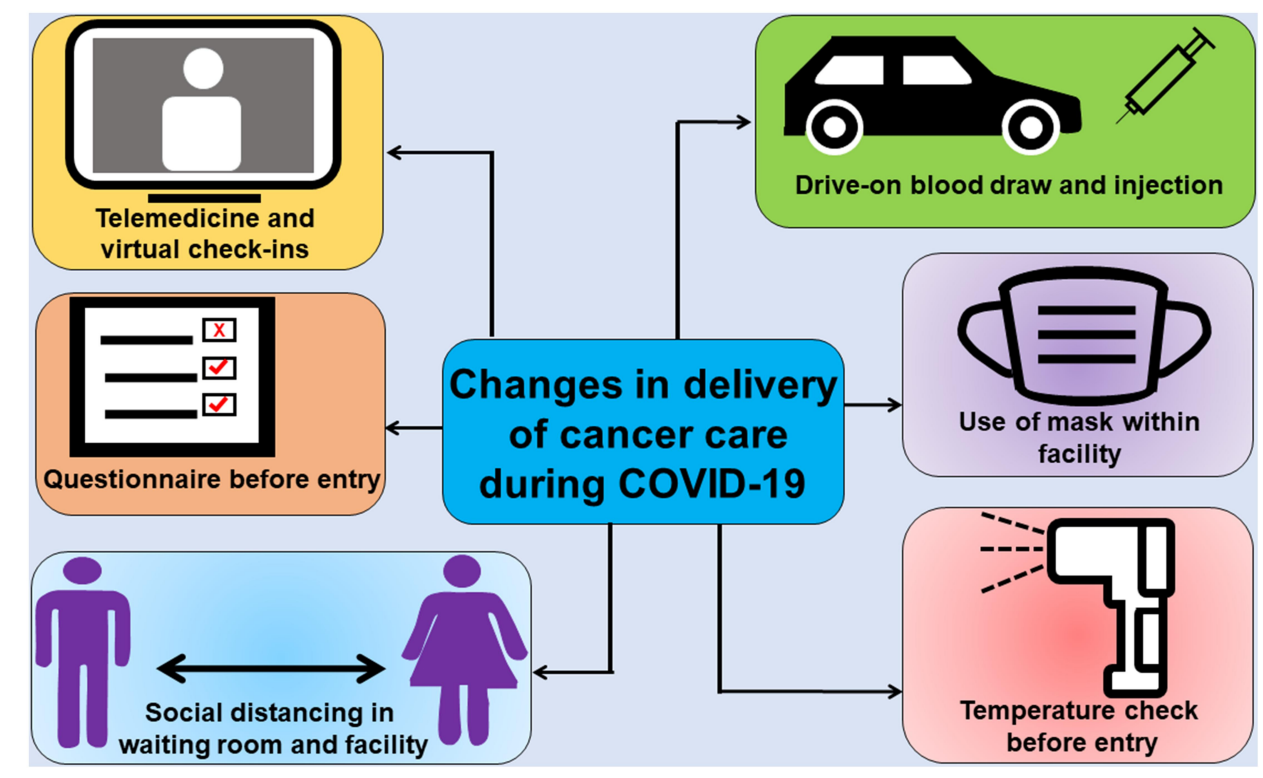

Figure 2 Effect of COVID-19 pandemic on the delivery of cancer care. Pandemic has severely impacted the delivery of cancer care. As a result, several adaptive changes have been made to protect both patients and health workers from SARS-CoV2 infection. These changes include new screening procedures such as temperature check and self-assessment questionnaire before entering the facility, enabling social distancing, mandatory use of masks in the work areas, drive through testing and injections, and virtual check-ins and telemedicine.

to receive the treatment they need without delay while less likely to suffer from health complications due to the virus infection. The number of daily new COVID-19 cases, hospitalization rates, and mortality have now begun to stabilize or decline in several states, attributed to stay-at-home orders and the use of masks at public places. However, the health care systems are keeping themselves ready for the possibility of new outbreaks and the second wave of infections. As the hospitals adapt to COVID-19 challenges, many new and advanced-stage cancer diagnoses and poor clinical outcomes are expected due to the delay in procedures. Furthermore, the disruption of treatment to reduce viral exposures could also turn counterproductive as patient 
survival is positively correlated with cancer. The new treatment strategies noted above can certainly help slow the spread of SARS-CoV-2 in OC patients, but not necessarily be the best available options for the treatment. Therefore, it is crucial to analyze each therapy's risk-benefit profile and other factors, including the patient's financial status and emergency services availability. There is also an urgent need for developing effective recovery plans that can focus on support services, particularly psychological support. By implementing new and effective COVID-19 management plans and developing creative ways to offer uninterrupted treatment to OC patients, we can achieve the goal of having active cancer survivors instead of passive victims.

\section{Funding}

This work was supported by the National Institutes of Health/National Cancer Institute [CA204801 and CA231925], and the University of South Alabama Mitchell Cancer Institute.

\section{Disclosure}

The authors report no conflicts of interest for this work.

\section{References}

1. Spiteri G, Fielding J, Diercke M, et al. First cases of coronavirus disease 2019 (COVID-19) in the WHO European Region, 24 January to 21 February 2020. Euro Surveill. 2020;25(9). doi:10.2807/15607917.ES.2020.25.9.2000178

2. Nicola M, O'Neill N, Sohrabi C, Khan M, Agha M, Agha R. Evidence based management guideline for the COVID-19 pandemic - Review article. Int J Surg. 2020;77:206-216. doi:10.1016/j.ijsu. 2020.04.001

3. Sohrabi C, Alsafi Z, O'Neill N, et al. World Health Organization declares global emergency: a review of the 2019 novel coronavirus (COVID-19). Int J Surg. 2020;76:71-76. doi:10.1016/j.ijsu.2020. 02.034

4. Coronavirus Cases; 2020. Available from: https://www.worldometers. info/coronavirus/. Accessed March 12, 2020.

5. Cascella M, Rajnik M, Cuomo A, Dulebohn SC, Di Napoli R. Features, evaluation, and treatment of Coronavirus (COVID-19). In: StatPearls. Treasure Island (FL): StatPearls Publishing; 2020.

6. Fagbule OF. 2019 Novel Coronavirus. Ann Ib Postgrad Med. 2019;17 (2):108-110.

7. Wu D, Wu T, Liu Q, Yang Z. The SARS-CoV-2 outbreak: what we know. Int J Infect Dis. 2020;94:44-48. doi:10.1016/j.ijid.2020.03.004

8. Ye ZW, Yuan S, Yuen KS, Fung SY, Chan CP, Jin DY. Zoonotic origins of human coronaviruses. Int J Biol Sci. 2020;16(10): 1686-1697. doi:10.7150/ijbs.45472

9. Karia R, Gupta I, Khandait H, Yadav A, Yadav A. COVID-19 and its Modes of Transmission. SN Compr Clin Med. 2020;1-4.

10. Grant MC, Geoghegan L, Arbyn M, et al. The prevalence of symptoms in 24,410 adults infected by the novel coronavirus (SARS-CoV2; COVID-19): a systematic review and meta-analysis of 148 studies from 9 countries. PLoS One. 2020;15(6):e0234765. doi:10.1371/journal.pone. 0234765
11. Emami A, Javanmardi F, Pirbonyeh N, Akbari A. Prevalence of underlying diseases in hospitalized patients with COVID-19: a systematic review and meta-analysis. Arch Acad Emerg Med. 2020;8(1):e35.

12. Mueller AL, McNamara MS, Sinclair DA. Why does COVID-19 disproportionately affect older people? Aging (Albany NY). 2020;12 (10):9959-9981. doi:10.18632/aging.103344

13. Siegel RL, Miller KD, Jemal A. Cancer statistics, 2020. CA Cancer J Clin. 2020;70(1):7-30. doi:10.3322/caac.21590

14. Deshmukh SK, Tyagi N, Khan MA, et al. Gemcitabine treatment promotes immunosuppressive microenvironment in pancreatic tumors by supporting the infiltration, growth, and polarization of macrophages. Sci Rep. 2018;8(1):12000. doi:10.1038/s41598-018$30437-2$

15. Gonzalez H, Hagerling C, Werb Z. Roles of the immune system in cancer: from tumor initiation to metastatic progression. Genes Dev. 2018;32(19-20):1267-1284. doi:10.1101/gad.314617.118

16. Kalathil SG, Thanavala Y. High immunosuppressive burden in cancer patients: a major hurdle for cancer immunotherapy. Cancer Immunol Immunother. 2016;65(7):813-819. doi:10.1007/s00262-016-1810-0

17. Ballard M, Bancroft E, Nesbit J, et al. Prioritising the role of community health workers in the COVID-19 response. BMJ Glob Health. 2020;5(6):e002550. doi:10.1136/bmjgh-2020-002550

18. Barach P, Fisher SD, Adams MJ, et al. Disruption of healthcare: will the COVID pandemic worsen non-COVID outcomes and disease outbreaks? Prog Pediatr Cardiol. 2020;59:101254. doi:10.1016/j. ppedcard.2020.101254

19. Perrone AM, De Palma A, De Iaco P. COVID-19 global pandemic: options for management of gynecologic cancers. The experience in surgical management of ovarian cancer in the second highest affected Italian region. Int J Gynecol Cancer. 2020;30(6):902. doi:10.1136/ ijgc-2020-001489

20. Chu KM, Smith M, Steyn E, Goldberg P, Bougard H, Buccimazza I. Changes in surgical practice in 85 South African hospitals during COVID-19 hard lockdown. S Afr Med J. 2020;110(9):916-919.

21. Kumar D, Dey T. Treatment delays in oncology patients during COVID-19 pandemic: a perspective. J Glob Health. 2020;10 (1):010367. doi:10.7189/jogh.10.010367

22. Sharma DC. Lockdown poses new challenges for cancer care in India. Lancet Oncol. 2020;21(7):884. doi:10.1016/S1470-2045(20) 30312-0

23. Tisdell CA. Economic, social and political issues raised by the COVID-19 pandemic. Econ Anal Policy. 2020;68:17-28. doi:10. 1016/j.eap.2020.08.002

24. Chudasama YV, Gillies CL, Zaccardi F, et al. Impact of COVID-19 on routine care for chronic diseases: a global survey of views from healthcare professionals. Diabetes Metab Syndr. 2020;14(5):965-967. doi:10.1016/j.dsx.2020.06.042

25. Gopalan HS, Misra A. COVID-19 pandemic and challenges for socio-economic issues, healthcare and National Health Programs in India. Diabetes Metab Syndr. 2020;14(5):757-759. doi:10.1016/j. dsx.2020.05.041

26. Nicola M, Alsafi Z, Sohrabi C, et al. The socio-economic implications of the coronavirus pandemic (COVID-19): a review. Int J Surg. 2020;78:185-193. doi:10.1016/j.ijsu.2020.04.018

27. Das PM, Bast Jr RC. Early detection of ovarian cancer. Biomark Med. 2008;2(3):291-303. doi:10.2217/17520363.2.3.291

28. Torre LA, Trabert B, DeSantis CE, et al. Ovarian cancer statistics, 2018. CA Cancer J Clin. 2018;68(4):284-296. doi:10.3322/caac. 21456

29. Doubeni CA, Doubeni AR, Myers AE. Diagnosis and management of ovarian cancer. Am Fam Physician. 2016;93(11):937-944.

30. Chien J, Poole EM. Ovarian cancer prevention, screening, and early detection: report from the 11th biennial ovarian cancer research symposium. Int $J$ Gynecol Cancer. 2017;27(Suppl 9):S20-S22. doi:10.1097/IGC.0000000000001118 
31. Chandra A, Pius C, Nabeel M, et al. Ovarian cancer: current status and strategies for improving therapeutic outcomes. Cancer Med. 2019;8(16):7018-7031. doi:10.1002/cam4.2560

32. Cortez AJ, Tudrej P, Kujawa KA, Lisowska KM. Advances in ovarian cancer therapy. Cancer Chemother Pharmacol. 2018;81(1): 17-38. doi:10.1007/s00280-017-3501-8

33. Indini A, Rijavec E, Ghidini M, Cattaneo M, Grossi F. Developing a risk assessment score for patients with cancer during the coronavirus disease 2019 pandemic. Eur J Cancer. 2020;135:47-50. doi:10.1016/j.ejca.2020.05.017

34. Indini A, Aschele C, Cavanna L, et al. Reorganisation of medical oncology departments during the novel coronavirus disease-19 pandemic: a nationwide Italian survey. Eur J Cancer. 2020;132:17-23. doi:10.1016/j.ejca.2020.03.024

35. Ramirez PT, Chiva L, Eriksson AGZ, et al. COVID-19 global pandemic: options for management of gynecologic cancers. Int J Gynecol Cancer. 2020;30(5):561-563. doi:10.1136/ijgc-2020-001419

36. Greer BE, Swensen RE, Gray HJ. Surgery for ovarian cancer: rationale and guidelines. J Natl Compr Canc Netw. 2004;2(6):561-568. doi:10.6004/jnccn.2004.0047

37. Smith CG, Davenport DL, Gorski J, et al. Clinical factors associated with longer hospital stay following ovarian cancer surgery. Healthcare (Basel). 2019;7(3). doi:10.3390/healthcare7030085.

38. Stewart JM, Tone AA, Jiang H, et al. The optimal time for surgery in women with serous ovarian cancer. Can J Surg. 2016;59(4):223-232. doi:10.1503/cjs.014315

39. Al-Jabir A, Kerwan A, Nicola M, et al. Impact of the Coronavirus (COVID-19) pandemic on surgical practice - Part 2 (surgical prioritisation). Int J Surg. 2020;79:233-248. doi:10.1016/j.ijsu.20 20.05 .002

40. Al-Jabir A, Kerwan A, Nicola M, et al. Impact of the Coronavirus (COVID-19) pandemic on surgical practice - Part 1. Int J Surg. 2020;79:168-179. doi:10.1016/j.ijsu.2020.05.022

41. Voices of women with ovarian cancer: the coronavirus pandemic and its impact; 2020. Available from: https://targetovariancancer.org.uk/ sites/default/files/2020-07/Voices\%20of\%20women\%20with\%20ovar ian $\% 20$ cancer $\% 20-\% 20$ the $\% 20$ coronavirus $\% 20$ pandemic $\% 20$ and $\%$ 20its\%20impact.pdf. Accessed 2020.

42. Frey MK, Ellis AE, Zeligs K, et al. Impact of the coronavirus disease 2019 pandemic on the quality of life for women with ovarian cancer. Am J Obstet Gynecol. 2020;223:725.e1-725.e9. doi:10.1016/j.ajog.20 20.06.049

43. Bogani G, Raspagliesi F. Minimally invasive surgery at the time of COVID-19: the OR staff needs protection. J Minim Invasive Gynecol. 2020;27(5):1221. doi:10.1016/j.jmig.2020.04.010

44. Bogani G, Apolone G, Ditto A, et al. Impact of COVID-19 in gynecologic oncology: a Nationwide Italian Survey of the SIGO and MITO groups. J Gynecol Oncol. 2020;31(6):e92. doi:10.3802/ jgo.2020.31.e92

45. Nakayama J, El-Nashar SA, Waggoner S, Traughber B, Kesterson J. Adjusting to the new reality: evaluation of early practice pattern adaptations to the COVID-19 pandemic. Gynecol Oncol. 2020;158 (2):256-261. doi:10.1016/j.ygyno.2020.05.028
46. Indini A, Rijavec E, Ghidini M, et al. Coronavirus infection and immune system: an insight of COVID-19 in cancer patients. Crit Rev Oncol Hematol. 2020;153:103059. doi:10.1016/j.critrevonc.2020.103059

47. Lei S, Jiang F, Su W, et al. Clinical characteristics and outcomes of patients undergoing surgeries during the incubation period of COVID-19 infection. EClinicalMedicine. 2020;21:100331. doi:10.10 16/j.eclinm.2020.100331

48. Liu Y, Song Y, Hu X, Yan L, Zhu X. Awareness of surgical smoke hazards and enhancement of surgical smoke prevention among the gynecologists. J Cancer. 2019;10(12):2788-2799. doi:10.7150/ jca.31464

49. Mowbray NG, Ansell J, Horwood J, et al. Safe management of surgical smoke in the age of COVID-19. Br J Surg. 2020. doi:10.1002/bjs.11679

50. Bogani G, Casarin J, Pinelli C, et al. Management of patients with ovarian cancer in the COVID-19 era. J Surg Oncol. 2020;122 (2):122-123. doi:10.1002/jso.26057

51. Bogani G, Signorelli M, Ditto A, Raspagliesi F. Surgical oncology at the time of COVID-19 outbreak. J Surg Oncol. 2020;122(2):115-116. doi:10.1002/jso.25975

52. Cooney E We have a job to do': cancer patients and their doctors carry on with clinical trials during Covid-19; 2020. Available from: https:/www.statnews.com/2020/06/16/cancer-patients-doctors-carryon-clinical-trials-during-covid-19/. Accessed 2020.

53. Crawford A, Haber L, Kelly MP, et al. A Mucin 16 bispecific T cell-engaging antibody for the treatment of ovarian cancer. Sci Transl Med. 2019;11:497. doi:10.1126/scitranslmed.aau7534

54. Kent DG, Knapp D, Kannan N. Survey says: “COVID-19 lockdown hits young faculty and clinical trials. Stem Cell Rep. 2020;15(1):1-5. doi:10.1016/j.stemcr.2020.06.010

55. Monk BJ, Coleman RL, Moore KN, et al. COVID-19 and ovarian cancer: exploring alternatives to intravenous (IV) therapies. Gynecol Oncol. 2020;158(1):34-36. doi:10.1016/j.ygyno.2020.04.703

56. Nassisi M, Audo I, Zeitz C, et al. Impact of the COVID-19 lockdown on basic science research in ophthalmology: the experience of a highly specialized research facility in France. Eye (Lond). 2020;34(7):1187-1188. doi:10.1038/s41433-020-0944-7

57. Rolston KV. Infections in cancer patients with solid tumors: a review. Infect Dis Ther. 2017;6(1):69-83. doi:10.1007/s40121-017-0146-1

58. Rusu RA, Sirbu D, Curseu D, et al. Chemotherapy-related infectious complications in patients with Hematologic malignancies. J Res Med Sci. 2018;23:68. doi:10.4103/jrms.JRMS_960_17

59. Walsh CS. Latest clinical evidence of maintenance therapy in ovarian cancer. Curr Opin Obstet Gynecol. 2020;32(1):15-21. doi:10.1097/ GCO.0000000000000592

60. Penson RT, Valencia RV, Cibula D, et al. Olaparib versus nonplatinum chemotherapy in patients with platinum-sensitive relapsed ovarian cancer and a germline BRCA1/2 mutation (SOLO3): a randomized phase III trial. J Clin Oncol. 2020;38(11):1164-1174. doi:10.1200/JCO.19.02745

61. Transforming futures for women with ovarian cancer. Available from: https://targetovariancancer.org.uk/sites/default/files/2020-07/ Pathfinder\%202016\%20-\%20Wales\%20report\%20\%28English\%29. pdf. 2020. Accessed 2020.

\section{Publish your work in this journal}

Cancer Management and Research is an international, peer-reviewed open access journal focusing on cancer research and the optimal use of preventative and integrated treatment interventions to achieve improved outcomes, enhanced survival and quality of life for the cancer patient.
The manuscript management system is completely online and includes a very quick and fair peer-review system, which is all easy to use. Visit http://www.dovepress.com/testimonials.php to read real quotes from published authors. 